\title{
An inverse problem in film/substrate indentation: extracting both the Young's modulus and thickness of films
}

\author{
Yin Zhang ${ }^{1,2} \cdot$ Feifei Gao ${ }^{1,2} \cdot$ Zhiyue Zheng $^{3} \cdot$ Zhihai Cheng $^{3}$
}

Received: 2 February 2018 / Revised: 1 March 2018 / Accepted: 26 March 2018 / Published online: 6 June 2018

(C) The Chinese Society of Theoretical and Applied Mechanics; Institute of Mechanics, Chinese Academy of Sciences and Springer-Verlag GmbH Germany, part of Springer Nature 2018

\begin{abstract}
In an indentation test, the effective Young's modulus of a film/substrate bilayer heterostructure varies with the indentation depth, a phenomenon known as the substrate effect. In previous studies investigating this, only the Young's modulus of the film was unknown. Once the effective Young's modulus of a film/substrate structure is determined at a given contact depth, the Young's modulus of the film can be uniquely determined, i.e., there is a one-to-one relation between the Young's modulus of the film and the film/substrate effective Young's modulus. However, at times it is extremely challenging or even impossible to measure the film thickness. Furthermore, the precise definition of the layer/film thickness for a two-dimensional material can be problematic. In the current study, therefore, the thickness of the film and its Young's modulus are treated as two unknowns that must be determined. Unlike the case with one unknown, there are infinite combinations of film thickness and Young's modulus which can yield the same effective Young's modulus for the film/substrate. An inverse problem is formulated and solved to extract the Young's modulus and thickness of the film from the indentation depth-load curve. The accuracy and robustness of the inverse problem-solving method are also demonstrated.
\end{abstract}

Keywords Indentation test · Film · Substrate · Inverse problem

\section{Introduction}

Two-dimensional (2D) transitional metal dichalcogenides (TMDs), such as molybdenum disulfide $\left(\mathrm{MoS}_{2}\right)$ and tungsten disulfide $\left(\mathrm{WS}_{2}\right)$, possess excellent optoelectronic and photocatalytic properties. The 2D material graphene is flexible and chemically stable, and exhibits excellent thermal and electrical conductivity. The properties of TMDs and graphene can be complementary to one another $[1,2]$. The hybrid materials/structures created by stacking combinations of 2D materials can provide new device architectures, which hold promise for energy conversion and storage [2].

Yin Zhang

zhangyin@1nm.imech.ac.cn

1 State Key Laboratory of Nonlinear Mechanics (LNM), Institute of Mechanics, Chinese Academy of Sciences, Beijing 100190, China

2 School of Engineering Science, University of Chinese Academy of Sciences, Beijing 100049, China

3 Department of Physics and Beijing Key Laboratory of Optoelectronic Functional Materials and Micro-Nano Devices, Renmin University of China, Beijing 100872, China
In a $2 \mathrm{D}$ heterostructure, the positive properties of each individual constituent material should be maintained or even amplified, while the negative properties should be mitigated [3]. For example, the graphene/ $\mathrm{MoS}_{2} /$ graphene trilayer heterostructure gives rise to the strong light-material interactions of enhanced photon absorption and electron-hole creation [1]; the heterostructure of the $\mathrm{MoS}_{2}$ monolayer, as a cavity sandwiched between $\mathrm{SiO}_{2}$ layers, results in strong light-material coupling and formation of cavity polaritons [4]; the $\mathrm{MoS}_{2} / \mathrm{WS}_{2}$ heterostructure enables construction of novel 2D devices for optoelectronics and light harvesting by facilitating ultrafast charge transfer [5]. The anisotropy of $2 \mathrm{D}$ materials is responsible for their unique properties [6]. The in-plane elasticity of 2D materials is an intrinsic property, while the out-of-plane elasticity is tunable [6]. Heterostructures created by stacking are also called van der Waals coupled layers [5]. Various ions and molecules can be accommodated between their layers [2,6], which, as a result, can change the $2 \mathrm{D}$ material properties. Characterizing the out-of-plane Young's modulus $\left(E_{\perp}\right)$ is essential for both fundamental understanding and practical application, since it relates to the thermal, electronic, tribological 
and optical properties of 2D materials. A work by Come et al. [6] showed that in an electrode composed of stacked 2D titanium carbide, the $E_{\perp}$ difference between the fully charged and discharged states can be as large as $15 \mathrm{GPa}$, and a strong correlation between $E_{\perp}$ and the cation content is thus established. A study by Gao et al. [7] showed that the $E_{\perp}$ of 2D graphene oxide depends on the number of water molecules intercalated between the layers. Various tests, including the uniaxial tensile test [8], bending test [9] and blister/bulge tests [10,11], are used to measure the in-plane Young's modulus. In contrast, indentation is among the very few techniques that can be used to measure $E_{\perp}$, and little is known about the $E_{\perp}$ of 2 D materials [7]. Two-dimensional materials are films with a thickness of a few atomic layers. One technical difficulty associated with indentation is the requirement of an indentation depth less than the film's interlayer distance [7]. In comparison, the above-referenced tests for the in-plane Young's modulus involve substantial deformation/deflection [8-11]. In a study by Gao et al. [7], the authors pioneered the use of atomic force microscopy (AFM) as an indentation tool to measure the $E_{\perp}$ of $2 \mathrm{D}$ materials. Even though $\mathrm{AFM}$ achieved a displacement resolution of $0.1 \AA$, they encountered difficulty when measuring the $E_{\perp}$ of the graphene monolayer on a silicon carbide ( $\mathrm{SiC}$ ) substrate with an interlayer distance of $3 \AA$ and indentation depth of $1 \AA$. This difficulty was referred to as a "complex convolution of effects and properties" [7] that resulted from the substrate effect.

In an indentation test of a film/substrate structure, the elastic field is not confined to the film itself, which is a long-ranged field that extends into the substrate. Therefore, the substrate properties influence the test outcome, a phenomenon known as the substrate effect. The presence of the in-plane stress causes the substrate to behave as an elastic foundation [12], which plays an important role in the transfer of stress between the film and substrate. As a result, the substrate effect, together with the in-plane pressure, has been shown to be an effective method for making the molecular crystal of carbon monoxide much harder on a $\mathrm{Cu}(001)$ substrate [13]. In order to measure the "film-only" properties, as a rule of thumb, the indentation depth should be limited to less than $10 \%$ of the film thickness [14]. However, the influence of the substrate on the film/substrate composite stiffness can be apparent even at very small indentation depths [15]. Therefore, this $10 \%$ rule is not always reliable [16]. When performing indentation tests on a stainless steel-coated copper substrate, Reed et al. [17] found that the substrate effect was already very significant at an indentation depth/coating thickness ratio of $2 \%$, while Bull [18] recommended a rule of $1 \%$. For the above case with an interlayer distance of $3 \AA$ and indentation depth of $1 \AA$ [7], the ratio is $33.33 \%$, and the substrate effect is very signif- icant or even dominant. Because shallow indentation results in inaccurate experimental measurements [16], the substrate effect must be considered in a film/substrate indentation test in order to extract the Young's modulus of the film [1317]. The variation in the effective Young's modulus of a film/substrate, which depends on the indentation depth, film thickness and Young's moduli of the film and substrate, has been studied intensively [13-19]. Several indentation models have been proposed for considering substrate effects, and these have been comprehensively reviewed by Bull [18] and Menčik et al. [19]. In all of the previous models [1319], the Young's modulus of the substrate was modeled as an elastic half-space with infinite thickness, and the film thickness was known. The only unknown in these models was the Young's modulus of the film, which can be obtained by a one-to-one function from data derived from the indentation test. Measurement of film thickness is often carried out separately in an indentation test. For example, the film thickness is measured by a profilometer [14,15], or by an AFM by performing a topographic scan of the sample [20]. Alternatively, the thickness can be precisely controlled during fabrication [16]. The accurate measurement of film thickness is difficult [19], especially when a film is thin. Furthermore, in some real applications such as coatings and substrates [18], soft samples [20] and plant cell walls (which are a three-dimensional (3D) nanocomposite consisting of several different layers) [21], measuring the coating/film/layer thickness is extremely difficult, if not impossible. For a 2D material, in addition to the measurement difficulty, simply defining the layer thickness can be tricky and controversial [22]. The interlayer distance can be obtained by discrete atomistic simulation [7,22]. However, for $2 \mathrm{D}$ materials, the layer thickness, as a concept of the continuum model, may or may not match the interlayer distance [22]. Furthermore, defining the layer thickness can be much more complicated when there are intermolecular bonds between the film and substrate layers [7,13,23]. This problem also pertains to macroscopic heterostructures. The film/layer (effective) thickness is not constant and varies as the indentation depth changes $[15,16,19]$. As will be discussed in more detail below, there is still disagreement among contemporary definitions of effective thickness [15,16,19].

In this study, film thickness and Young's modulus are treated as two unknowns which are solved as an inverse problem. Three different contact models and two models of the substrate effect are presented. Different contact models produce different load-indentation depth curves, from which the effective Young's modulus of the film/substrate composite is extracted. The effective Young's modulus of the composite, which varies with the indentation depth, is also determined by the substrate effect model. Once the effective Young's modulus is substituted into the substrate effect model, an inverse problem can be formulated. 
Although different substrate effect models lead to different inverse problem formulations, it is shown that the difference between these two models is negligible. The contact model of continuum mechanics is shown to be effectively applied in the $E_{\perp}$ measurement of 2D materials [7]. In the measurement of 10-layer graphene with a total interlayer distance (or thickness) of $30 \AA$, the indentation depth is $2 \AA$ and the SiC substrate is implicitly assumed to have no impact [7]. The ratio of the indentation depth to thickness is $2: 30 \approx 6.7 \%$; the graphene $E_{\perp} \approx 35 \mathrm{GPa}$ and $\mathrm{SiC}$ $E_{\perp} \approx 400 \mathrm{GPa}$ [7]. As will later be shown, the substrate effect is more pronounced at the initial indentation stage. When there is a large modulus difference between graphene and $\mathrm{SiC}$, the substrate effect is very significant, even with a small depth-to-thickness ratio of $6.7 \%$. Furthermore, the interlayer distance was shown by atomistic simulation to be dependent on the rate of water molecule intercalation between layers [7], which varies over a large range and is responsible for the $E_{\perp}$ variation. With this inverse problemsolving method, the $E_{\perp}$ of a $2 \mathrm{D}$ material can be more accurately obtained by considering the substrate effect, without measuring/computing or defining its layer thickness. The substrate effect is present in the indentation test of any sample of finite thickness on a substrate [20]. The film/substrate bilayer structure is simply a special type of heterostructure. Characterizing the mechanical properties of a layer on a base has long been of technical importance [24]. The inverse problem-solving method presented here essentially determines the Young's modulus and thickness of a film with the substrate effect. Therefore, in addition to the film/substrate bilayer, the method can be applied to general scenarios, such as the indentation of soft samples (gel, tissue, cell and polymer, etc.) [20,25] and biomass [21]. Furthermore, for experimentalists, approaches "solely relying on data from one or two very shallow indents" are preferred when determining the Young's modulus of a film/coating [17]. Here the inverse problem is based on models of elastic contact mechanics and is solved by obtaining two sets of indentation data.

\section{Model development}

The Hertz contact model [26] is given as follows:

$P=\frac{4 E^{*} a^{3}}{3 R}$

where $P$ is the indentation load and $a$ is the contact radius, $1 / R=1 / R_{i}+1 / R_{f}\left(R_{i}\right.$ : indenter radius and $R_{f}$ : the film radius of curvature). $E^{*}$ is the reduced modulus of the specimen/indenter system, which is given as

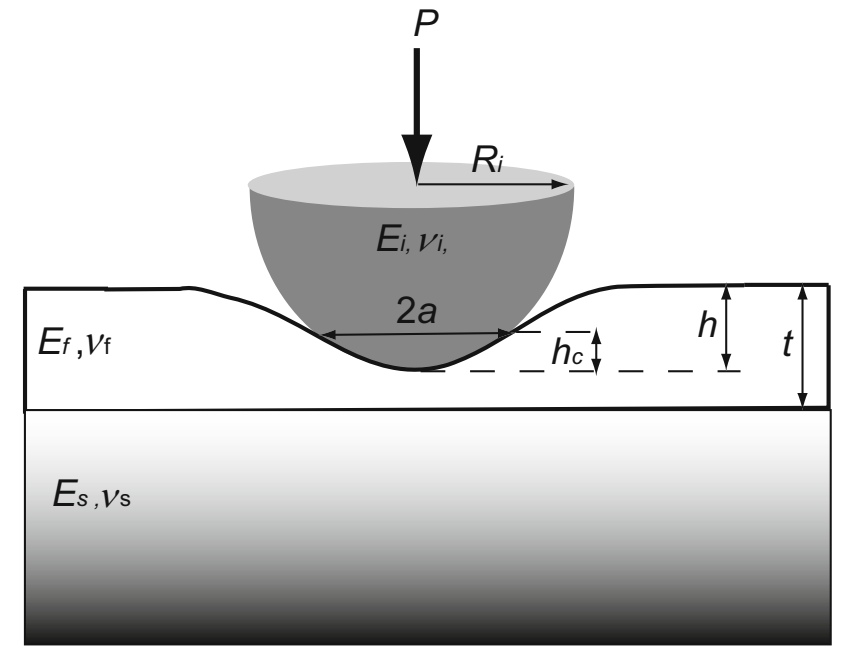

Fig. 1 Schematic of the penetration of an indenter into a film/substrate structure. The indenter has a radius of $R_{i}$, Young's modulus of $E_{i}$ and Poisson ratio of $v_{i} . t$ is the film thickness; $h_{c}$ and $a$ are the contact depth and radius, respectively. $P$ is the indentation load and $h$ is the indenter displacement. $E_{f}, v_{f}$ and $E_{s}, v_{s}$ are the Young's moduli and Poisson ratios of the film and substrate, respectively

$\frac{1}{E^{*}}=\frac{1}{E^{\prime}}+\frac{1}{E_{i}^{\prime}}$.

Here, $E^{\prime}$ is the reduced modulus of the specimen. As shown in Fig. 1, the specimen is a film/substrate structure, and the $E^{\prime}$ expression of the film/substrate structure will be discussed later in detail. $E_{i}^{\prime}=E_{i} /\left(1-v_{i}^{2}\right)$ is the reduced modulus of the indenter, which is also its plane-strain modulus [16]. Here, $E_{i}$ and $v_{i}$ are the Young's modulus and Poisson ratio of the indenter, respectively. The reduced modulus concept conveys the fact that the stress state in the contact zone is triaxial, with a high degree of hydrostatic pressure due to lateral constraints [19].

The Derjaguin-Muller-Toporov (DMT) contact model [27] is expressed as follows:

$P=\frac{4 E^{*} a^{3}}{3 R}-2 \pi R \gamma$

where $\gamma$ is the work of adhesion between the indenter and film surfaces. Compared with the Hertz model, the DMT model is offset by a tensile force of $-2 \pi R \gamma$, which is induced by the attractive surface/adhesion force outside the contact zone [28]. This tensile force of $-2 \pi R \gamma$ is also the pull-off force of the DMT model as the contact radius $a$ approaches zero. One remarkable feature of this pull-off force is its independence on the elastic moduli of contacting bodies, which is effectively used to determine the work of adhesion $(\gamma)$ [7].

The Johnson-Kendall-Roberts (JKR) contact model [29] is expressed as follows: 
$P=\frac{4 E^{*} a^{3}}{3 R}-2 a^{3 / 2} \sqrt{2 \pi E^{*} \gamma}$

In both the Hertz and DMT models, compressive stress occurs only inside the contact zone [28]. In contrast, both compressive and tensile stresses exist inside the contact zone of the JKR model [29]. The tensile stress of the JKR model inside the contact zone is induced by the work of adhesion [29], which is responsible for the $-2 a^{3 / 2} \sqrt{2 \pi E^{*} \gamma}$ term [30]. The applicability range of the above three contact models can be determined by the dimensionless Tabor number of $\mu=\left[R \gamma^{2} /\left(E^{* 2} z_{o}^{3}\right)\right]^{1 / 3}\left(z_{o}\right.$ : equilibrium separation of atoms) [31]. Because the JKR model assumes that a "neck" forms around the contact zone, the physical meaning of the Tabor number is the ratio of (the order of) "neck" height to $z_{o}$ [28]. If the neck height is large compared with $z_{o}$ (the large Tabor number scenario, say, $\mu>5$ [32]), the surface interaction force outside the contact area is small enough to be ignored, and this feature defines the JKR model [28]. Similarly, if the neck height is small compared with $z_{o}$ (the small Tabor number scenario, say, $\mu<0.1$ [32]), the surface interaction force outside the contact area is significant, and this scenario corresponds to the DMT model. By examining the definition of $\mu$, we can conclude that the JKR model applies when the work of adhesion and radii of contacting bodies are large and their Young's moduli are small. The DMT model follows the opposite trend; it is applicable when the work of adhesion and radii of contacting bodies are small and their Young's moduli are large. One requisite for application of both the JKR and DMT is that the external load $P$ is relatively small compared with the adhesion force. If $P$ is large (say, $P /(\pi R \gamma)>100[32]$ ), the effect of the adhesion force is too small and the Hertz model applies.

Equations (1), (3) and (4) present the $a-P$ curves. However, the quantity measured by a depth-sensing indentation instrument is the indentation depth $(h)$ rather than the contact radius $(a)$ [13,19,31-36]. The following relation holds for the contact depth $\left(h_{c}\right)$ and radius $(a)$ for a spherical indenter $[37,38]$

$h_{c}= \begin{cases}\frac{a^{2}}{R} & (\text { Hertz, DMT }), \\ \frac{a^{2}}{R}-\sqrt{\frac{2 \pi R \gamma}{E^{*}}} & (\mathrm{JKR}) .\end{cases}$

As shown in Fig. $1, h_{c}$ and $h$ are usually different [14,33]. In the sink-in scenario shown in Fig. $1, h_{c}<h$, the true contact depth $\left(h_{c}\right)$ is thus overestimated if the measured quantity $h$ is used as the contact depth, while in the pile-up scenario, as shown in Ref. [39], $h_{c}>h$ and the true contact depth is thus underestimated. Sink-in is due to the elastic deformation, and pile-up can only be formed by plastic deformation [36,39]. In general, a hard film on a soft substrate tends to sink in when indented, whereas a soft film on a hard substrate tends to pile up [14]. For simplicity, in this study, $h=h_{c}$ is assumed, which causes an error. This error will be discussed later, along with other sources of error.

The contact stiffness $(K)$ is obtained by taking the derivative of $\mathrm{d} P / \mathrm{d} h_{c}$, as follows:

$$
\begin{aligned}
K & =\frac{\mathrm{d} P}{\mathrm{~d} h_{c}}=\frac{\mathrm{d} P / \mathrm{d} a}{\mathrm{~d} h_{c} / \mathrm{d} a} \\
& = \begin{cases}2 E^{*} a=2 E^{*} \sqrt{R h_{c}} & (\text { Hertz, DMT) } \\
\frac{4 E^{*} a^{2} / R-3 \sqrt{2 \pi a \gamma}}{2 a / R-\sqrt{\pi \gamma /\left(2 E^{*} a\right)}} & (\mathrm{JKR}) .\end{cases}
\end{aligned}
$$

Clearly, when $\gamma=0, \mathrm{~d} P / \mathrm{d} h_{c}$ of the JKR model recovers that of the Hertz and DMT models. Equation (6) is derived from the elastic contact models, and thus the plastic effect is not considered. Because of the large indentation force, plastic deformation often occurs in the loading stage $[14,33,34]$. Although the $h-P$ curve and $K$ are extracted from the data of the unloading stage [14,33,34], which is (assumed to be) elastic, the influence of plastic deformation cannot be fully excluded. In addition to plasticity, other irreversible processes such as microcracking and macroscopic fracture can cause the indentation data to deviate significantly from data predicated on the elastic contact mechanics, and the interpretation of indentation data can therefore be extremely difficult [17]. The modified version of Eq. (6) is as follows [36]:

$$
K=C \frac{2}{\sqrt{\pi}} E^{*} \sqrt{S}
$$

where $C$ is a dimensionless correction factor and $S$ is the projected contact area. $C$ is (mainly) dependent on the indenter geometry. For example, $C=1$ is used for a spherical indenter, $C=1.012$ is used for a square indenter (punch) and $C=1.034$ is used for a triangular indenter [34]. The projected contact area is $S=\pi a^{2}$, and the $h_{c}-a$ relation is given in Eq. (5). However, due to the sink-in or pile-up effect, $h_{c}$ can be difficult to obtain from the measured data of $h$, making the proper estimation of $S$ difficult [40]. Clearly, when $C=1$, Eq. (7) recovers Eq. (6) of the Hertz and DMT models.

In the indentation tests, $P, h, R$ and $\gamma$ are the measured quantities. The contact radius $a$ is calculated from Eq. (5). The contact stiffness $K=K(a)$ or $K=K(h)$, which varies with contact radius/depth, is calculated from the $h-P$ curve. Mathematically, in the contact models (Eqs. (1), (3) and (4)) or the contact stiffness equations (Eqs. (6) and (7)), $E^{*}$ is the only unknown, which can be easily determined by one of those equations. In a real application, the measured pull-off forces, which determine the work of adhesion $(\gamma)$, fluctuate significantly among different tests [7]. This fluctuation directly impacts the explanation of the $a-P$ curves provided by the DMT and JKR models, which can result in significant errors when the indentation is shallow. In contrast, the pull-off force measurement has much less influence on the 
contact stiffness. In fact, it has no influence on the Hertz or DMT model, as seen in Eq. (6). For a shallow indentation, using the contact stiffness model of Eqs. (6) or (7) to extract $E^{*}$ is recommended [7]. Once $E^{*}$ is found, $E^{\prime}$ is determined from Eq. (2) as follows:

$E^{\prime}=\frac{E^{*} E_{i}^{\prime}}{E_{i}^{\prime}-E^{*}}$

Here, the indenter reduced modulus of $E_{i}^{\prime}$ is a known quantity, while $E^{\prime}=E^{\prime}\left(E_{f}^{\prime}, E_{s}^{\prime}, t, a\right)$ or $E^{\prime}=E^{\prime}\left(E_{f}^{\prime}, E_{s}^{\prime}, t, h\right)$ is a function of the reduced moduli of the film and substrate $\left(E_{f}^{\prime}\right.$ and $\left.E_{s}^{\prime}\right)$, the film thickness $(t)$ and the contact radius or depth $(a$ or $h)$. There are several function forms for $E^{\prime}=$ $E^{\prime}\left(E_{f}^{\prime}, E_{s}^{\prime}, t, a\right)$. However, a systematic study by Menčik et al. [19] shows that only two function forms can properly fit the experimental data. One is the exponential function form given as

$E^{\prime}=E_{s}^{\prime}+\left(E_{f}^{\prime}-E_{s}^{\prime}\right) \mathrm{e}^{-\Lambda a / t}$,

where $\Lambda$ is a positive fitting parameter, which can be obtained from the experimental data by the least squares method [19]. The other form is as follows form [41]:

$E^{\prime}=E_{s}^{\prime}+\left(E_{f}^{\prime}-E_{s}^{\prime}\right) \phi_{g}(x)$

where $x=a / t$ and $\phi_{g}(x)$ is referred to as Gao's function by Menčik et al. [19], which was originally developed for the shear modulus [41] and is given as

$$
\begin{aligned}
\phi_{g}(x)= & \frac{2}{\pi} \arctan \frac{1}{x} \\
& +\frac{1}{2 \pi(1-v)}\left[(1-2 v) \frac{\ln \left(1+x^{2}\right)}{x}-\frac{x}{1+x^{2}}\right] .
\end{aligned}
$$

Here, $v$ is the effective Poisson ratio, which is a function of the contact radius and film thickness, i.e., $v=v(a / t)$. As the contact radius/depth ( $a$ or $h$ ) increases, the $v$ value changes from the film's Poisson ratio $\left(v_{f}\right)$ to that of the substrate $\left(v_{s}\right)$ [41]. However, $E^{\prime}$ as given in Eq. (10) is very insensitive to the choice of $v$ [41], and Menčik et al. [19] suggested simply using $v_{f}$ for $v$. Because $\phi_{g}(x)$ is analytically derived [41], one outstanding characteristic of this function is that it lacks a fitting parameter. Both $\mathrm{e}^{-\Lambda x}$ and $\phi_{g}(x)$ monotonically decrease from 1 to 0 as $x$ increases from 0 to $\infty$, which indicates that the substrate effect is enhanced with increasing contact radius/depth. As a result, the $E^{\prime}$ value changes from $E_{f}^{\prime}$ to $E_{s}^{\prime}$. There are three implicit assumptions here. One is that the substrate is relatively thick in comparison to the indentation depth and the film thickness, and it therefore behaves as an elastic half-space. The second assumption is that the film and substrate are perfectly bound to ensure continuity at the interface for both the displacements and the stress components which are perpendicular to the interface [42]. Finally, it is assumed that $t$ is fixed as the film thickness without indentation. However, as indentation progresses, the (effective) film thickness may vary. For example, $\mathrm{Li}$ and Vlassak [16] argued that $t$ decreases during the indentation process because of the highly hydrostatic stress state under an indenter, which causes the indented materials to become more dense, thus leading to local thinning. According to Menčik et al. [19], the reason for the decrease in $t$ was that the indenter occupies the film volume, while Lim et al. [15] argued that $t$ can either increase due to pile-up or decrease due to sink-in. Because local thinning of the film is not uniform, precisely defining the effective film thickness can be problematic [16].

In Eqs. (9) and (10), the reduced modulus of the substrate $E_{s}^{\prime}$ is a known quantity, and $E^{\prime}$ is measured. If the film thickness $t$ is also a known quantity, the reduced modulus of the film $E_{f}^{\prime}$ is the only unknown, which can be simply determined as $E_{f}^{\prime}=\left(E^{\prime}-E_{s}^{\prime}\right) \mathrm{e}^{\Lambda a / t}+E_{s}^{\prime}$ from Eq. (9) or $E_{f}^{\prime}=\left(E^{\prime}-E_{s}^{\prime}\right) / \phi(x)+E_{s}^{\prime}$ from Eq. (10). However, there is no simple and accurate method for the determination of the true film thickness [19], especially when the film thickness is very small. In 2D materials, the interlayer distance, which is several angstrom, varies due to the intercalation of water between the layers and the presence of a buffer layer [7]. In such a scenario, measuring or computing the (effective) layer thickness in continuum mechanics is extremely difficult, if not impossible. Similarly, for sub-micron-scale biological tissue or soft samples on a substrate, the measurement of thickness poses a technical challenge [20]. Now an inverse problem arises: how to determine $E_{f}^{\prime}$ without knowing $t$ in the film/substrate indentation test. Mathematically, $E_{f}^{\prime}$ and $t$ are now two unknowns. Unlike the previous scenario with a single unknown where $E_{f}^{\prime}$ is uniquely determined as a function, for given $E^{\prime}, E_{s}^{\prime}$ and $a$, there are infinite combinations of $E_{f}^{\prime}$ and $t$ that can satisfy Eq. (9) or (10). Before formulating and solving the inverse problem, the following quantities are introduced for the nondimensionalization scheme:

$$
\begin{aligned}
A & =\frac{a}{a_{c}}, \quad F=\frac{P}{P_{c}}, \\
\alpha_{1} & =\frac{E^{*}}{E_{i s}^{\prime}}, \quad \alpha_{2}=\frac{E_{f}^{\prime}}{E_{i s}^{\prime}}, \quad \alpha_{3}=\frac{E_{s}^{\prime}}{E_{i s}^{\prime}}, \quad \beta_{1}=\frac{E_{i}^{\prime}}{E_{i s}^{\prime}}, \\
T & =\frac{t}{a_{c}}, \quad x=\frac{a}{t}=\frac{A}{T} .
\end{aligned}
$$

Here, $E_{i s}^{\prime}=E_{i}^{\prime} E_{s}^{\prime} /\left(E_{i}^{\prime}+E_{s}^{\prime}\right)$ (i.e., $\left.1 / E_{i s}^{\prime}=1 / E_{i}^{\prime}+1 / E_{s}^{\prime}\right)$ is the reduced modulus if the indenter is in contact with the substrate; $a_{c}=\left[9 \pi \gamma R /\left(8 E_{i s}^{\prime}\right)\right]^{1 / 3}$ is the JKR pull-off radius if the indenter is in contact with the substrate and with the 
work of adhesion of $\gamma ; P_{c}=3 \pi R \gamma / 2$ and $-P_{c}$ is the (tensile) JKR pull-off force [29]. Physically, $\alpha_{1}$ is the dimensionless reduced modulus of the specimen/indenter system, and here the specimen is the film/substrate structure; $\alpha_{2}$ and $\alpha_{3}$ are the dimensionless reduced modulus of the film and substrate, respectively. $\beta_{1}$ is the dimensionless reduced modulus of the indenter, $T$ is the dimensionless film thickness, and $x$ is the ratio of the contact radius to the film thickness. The Hertz and DMT models of Eqs. (1) and (3) are now nondimensionalized as follows:

$$
\begin{array}{ll}
\text { Hertz : } & F=\alpha_{1} A^{3}, \\
\text { DMT : } & F=\alpha_{1} A^{3}-\frac{4}{3} .
\end{array}
$$

The JKR model is now nondimensionalized as follows:

$$
F=\alpha_{1} A^{3}-2 A^{3 / 2} \sqrt{\alpha_{1}} .
$$

In an indentation test, $F$ and $A$ are the known quantities, and $\alpha_{1}$ can thus be extracted from Eq. (13) for the Hertz and DMT models as follows:

$\alpha_{1}= \begin{cases}\frac{F}{A^{3}} & (\text { Hertz }), \\ \frac{F+4 / 3}{A^{3}} & (\text { DMT }) .\end{cases}$

Keep in mind that $\alpha_{1}$ is a function of the reduced Young's moduli of the indenter, film and substrate, work of adhesion, film thickness and the contact radius or contact depth, i.e., $\alpha_{1}=\alpha_{1}\left(E_{i}^{\prime}, E_{f}^{\prime}, E_{s}^{\prime}, \gamma, t, a\right)$ or $\alpha_{1}=\alpha_{1}\left(E_{i}^{\prime}, E_{f}^{\prime}, E_{s}^{\prime}, \gamma\right.$, $t, h)$. For the JKR model, $\alpha_{1}$ is derived from Eq. (14) as follows:

$\alpha_{1}= \begin{cases}\frac{F+2+2 \sqrt{F+1}}{A^{3}} & \left(A>A_{c}^{*}\right), \\ \frac{F+2-2 \sqrt{F+1}}{A^{3}} & \left(A \leq A_{c}^{*}\right) .\end{cases}$

Here, $A_{c}^{*}$ is the dimensionless pull-off radius as the indenter is in contact with the film/substrate. As the above $a_{c}$ is defined as the pull-off contact radius as if the indenter is in contact with the substrate, $A_{c}^{*}=1$ only when $\alpha_{1}=1$ (i.e., the substrate-only case). $A_{c}^{*}$ varies as the film properties change. Equation (8) is now nondimensionalized as

$\frac{E^{\prime}}{E_{i s}^{\prime}}=\frac{\alpha_{1} \beta_{1}}{\beta_{1}-\alpha_{1}}$

Equations (9) and (10) can be nondimensionalized together as follows:

$\frac{E^{\prime}}{E_{i s}^{\prime}}=f=\alpha_{3}+\left(\alpha_{2}-\alpha_{3}\right) \phi(x)$,

where $\phi(x)=\mathrm{e}^{-\Lambda x}$ corresponds to the exponential function and $\phi(x)=\phi_{g}(x)$ is Gao's function. Here, $x=A / T$.
By equating Eqs. (17) and (18), the following equation is derived:

$$
\begin{aligned}
\mathcal{F}\left(\beta_{1}, \alpha_{1}, \alpha_{3}, A, \alpha_{2}, T\right)= & \frac{\alpha_{1} \beta_{1}}{\beta_{1}-\alpha_{1}}-\alpha_{3} \\
& -\left(\alpha_{2}-\alpha_{3}\right) \phi(x)=0 .
\end{aligned}
$$

\section{Results and discussion}

In this study, the indenter and substrate are both silicon with a Young's modulus of $169 \mathrm{GPa}$ and a Poisson ratio of 0.27 ; the indenter radius is $R_{i}=114 \mathrm{~nm}$ and the film/substrate are flat, which leads to $R=114 \mathrm{~nm}$ [7]. Because water intercalation due to the relative humidity can significantly impact the work of adhesion $(\gamma)$ [7], the value of $\gamma$ calculated from the measured pull-off forces using the DMT model varies over a large range, from $\gamma=0.03 \mathrm{~N} / \mathrm{m}$ to $\gamma=1 \mathrm{~N} / \mathrm{m}$. Here, $\gamma=0.1 \mathrm{~N} / \mathrm{m}$ is taken as a typical value, and the pull-off radius is calculated as $a_{c}=3.69 \mathrm{~nm}$. The film thickness is fixed as $t=1.5 a_{c}$. In summary, the reduced Young's moduli of the indenter and substrate are fixed, which leads to $\beta_{1}=\alpha_{3}=2$; the fixed film thickness leads to $T=1.5$. The $A-F$ of the force-contact radius curves as given by the Hertz, DMT and JKR models of Eqs. (13) and (14) are plotted in Fig. 2. The film is germanium with $E_{f}=138 \mathrm{GPa}$ and $v_{f}=0.26$, which gives $\alpha_{2}=1.6239$. In Fig. 2, both Hertz and DMT models predict stable contact until separation (i.e., $A=0$ ). In contrast, the JKR model predicts an unstable snap-through behavior of pull-off at $A_{c}$ [29]. Since

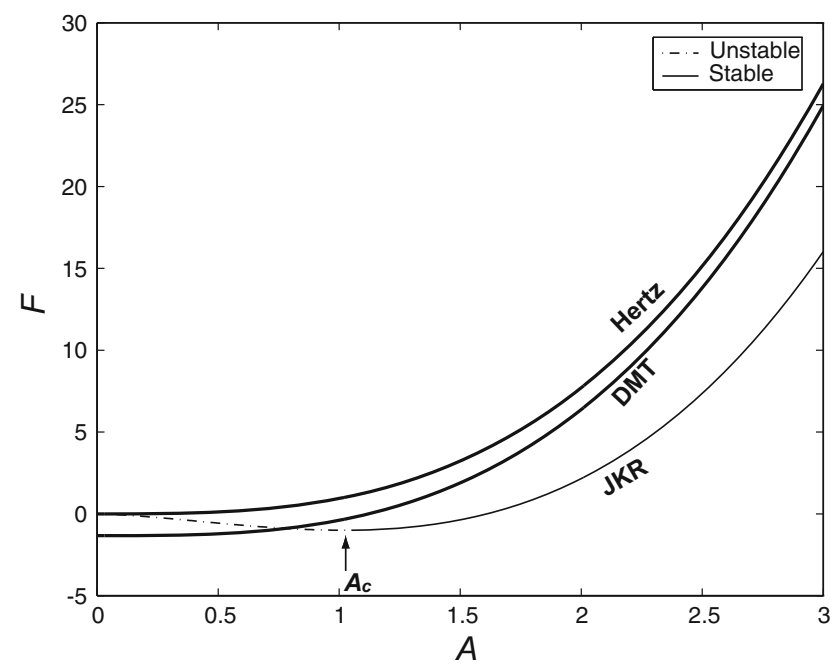

Fig. $2 A-F$ curves of the $\mathrm{Ge} / \mathrm{Si}$ heterostructure with $\alpha_{2}=1.62$ as given by three different contact models. For the JKR model, the indenter will experiences a snap-through behavior of pull-off at $A_{c}$, and thus $A<A_{c}$ is an unstable path, which is marked with a dashed line, while both DMT and Hertz remain stable 


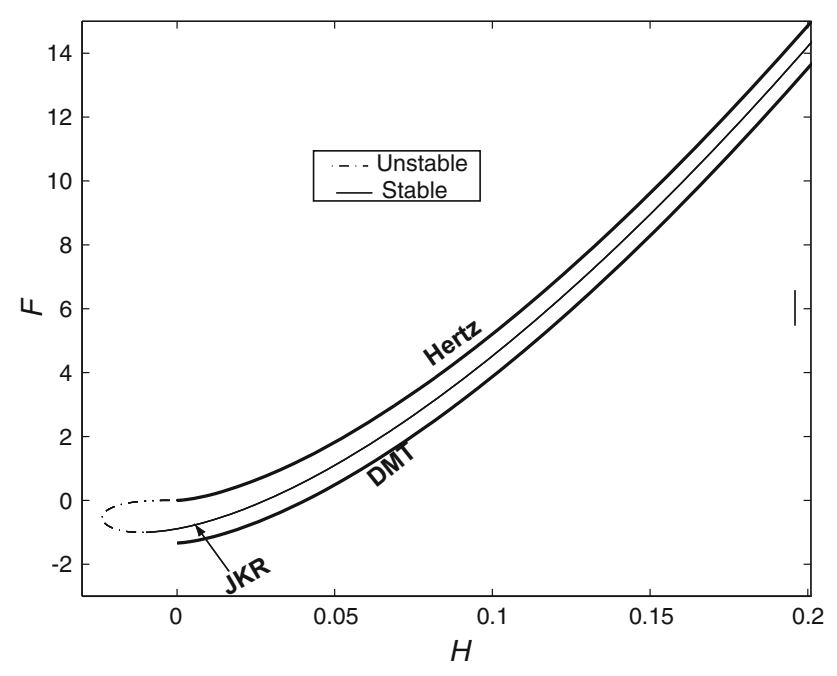

Fig. $3 H-F$ curves of the $\mathrm{Ge} / \mathrm{Si}$ heterostructure with $\alpha_{2}=1.62$ as given by three different contact models

this scenario involves a soft film on a hard substrate, $A_{c}$ is found to be $A_{c}=1.02$.

By assuming $h=h_{c}$, Eq. (5) is now nondimensionalized as the following:

$H=\frac{h}{a_{c}}= \begin{cases}d_{1} A^{2} & (\text { Hertz, DMT }) \\ d_{1} A^{2}-\sqrt{d_{2} A} & (\mathrm{JKR})\end{cases}$

Here, $d_{1}$ and $d_{2}$ are two dimensionless parameters. $d_{1}=$ $a_{c} / R$ has a fixed value; $d_{2}=2 \pi \gamma /\left(E^{*} a_{c}\right)=2 \pi \gamma /\left(\alpha_{1} E_{i s}^{\prime} a_{c}\right)$ is a variable quantity, as $\alpha_{1}$ varies with the indentation depth (or contact radius). In conjunction with Eqs. (13), (14) and (20), the $H-F$ curves of the three contact models are plotted in Fig. 3. Again, there is an unstable branch for the JKR model. As shown in Eq. (6), the contact stiffness is extracted from the $\mathrm{d} P / \mathrm{d} h(\mathrm{~d} F / \mathrm{d} H)$ curve. The three $H-F$ curves of the different contact models, as shown in Fig. 3, are (almost) parallel when $H>0.15$, which means they share nearly the same contact stiffness. Therefore, if the contact stiffness of Eq. (6) is used to extract $E^{*}$, the difference caused by the different contact models is only obvious when the contact load (or contact depth/radius) is very small. For this reason, the Hertz model is found to be very accurate in the indentation test of 2D materials [7]. It is clear from Figs. 2 and 3 that different contact models result in the $H-F$ curves. In the practical application of an indentation test, the adhesion and Young's modulus of the film are measured. Therefore, the Tabor number is unknown, and the contact model must be chosen. The use of an improper contact model will result in an error, which is discussed later in detail.

Figure 4 examines the influence of different films on the $H-F$ curves of the DMT model. In Fig. 4, there are three different films: $E_{f}=400 \mathrm{GPa}$ and $v_{f}=0.14$ for SiC [7], $E_{f}=169 \mathrm{GPa}$ and $v_{f}=0.27$ for silicon $(\mathrm{Si})$, and $E_{f}=138$

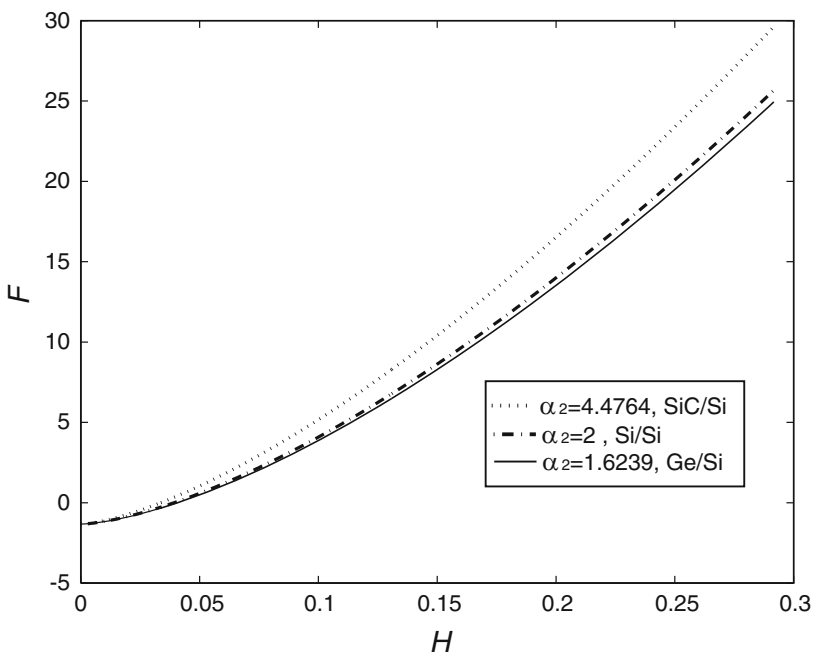

Fig. $4 H-F$ curves of the DMT models with different $\alpha_{2}$. Here $\alpha_{2}=$ 4.4764 is for the $\mathrm{SiC} / \mathrm{Si}, \alpha_{2}=1.6239$ for $\mathrm{Ge} / \mathrm{Si}$ and $\alpha_{2}=2$ for the $\mathrm{Si}$ substrate only

$\mathrm{GPa}$ and $v_{f}=0.26$ for germanium (Ge), which form the $\mathrm{SiC} / \mathrm{Si}, \mathrm{Si} / \mathrm{Si}$ and $\mathrm{Ge} / \mathrm{Si}$ types of film/substrate structures. According to the definition of Eq. (12), $\alpha_{2}=4.4764$ for $\mathrm{SiC} / \mathrm{Si}, \alpha_{2}=2$ for $\mathrm{Si} / \mathrm{Si}$, and $\alpha_{2}=1.6239$ for $\mathrm{Ge} / \mathrm{Si}$. The $\mathrm{Si} / \mathrm{Si}$ case with $\alpha_{2}=2$ is the de facto substrate-only case. The $F-H$ curve with $\alpha_{2}=4.4764$ of $\mathrm{SiC} / \mathrm{Si}$ is the upper one, and that with $\alpha_{2}=1.6239$ of $\mathrm{Ge} / \mathrm{Si}$ is the lower one. Because $\alpha_{2}=4.4764$ is the hard film case, a larger indentation force is required for a given contact depth, and because $\alpha_{2}=1.6239$ is the soft film case, a smaller indentation force is required. The effective reduced Young's modulus of the film/substrate structure varies with the contact depth, which is embodied in Eq. (18). Figure 5 plots the change in the effective modulus as $H$ changes. In Fig. 5, $f=E^{\prime} / E_{i s}^{\prime}=\alpha_{3}+\left(\alpha_{2}-\alpha_{3}\right) \phi(x)$ and $\phi(x)=\phi_{g}(x)$ is used. Because $\phi_{g}(x)$ is very insensitive to the effective Young's modulus $v$ [41], $v=0.3$ is taken. $\alpha_{2}=2$ remains a straight line. Both the $\alpha_{2}=4.4764$ and $\alpha_{2}=1.6239$ curves approach that of $\alpha_{2}=2$ as $H$ increases. As the indentation depth increases, the substrate plays an increasingly important role and eventually becomes dominant, which is the physical mechanism responsible for the approaching behavior. In addition, both the $\alpha_{2}=4.4764$ and $\alpha_{2}=1.6239$ curves experience rapid change when $H$ is small, which physically means that even a small indentation depth can cause a significant change in the effective reduced Young's modulus of the film/substrate structure. As mentioned above, the rule of thumb for measuring the "filmonly" properties is to limit the indentation depth to less than $10 \%$ of the film thickness [14]. At $H=0.15$, which is $10 \%$ of the film thickness $(T=1.5), f=E^{\prime} / E_{i s}^{\prime}=1.8676$ is the $15.01 \%$ increase for the $f=1.6239$ of $\mathrm{Ge} / \mathrm{Si}$ at $H=0$. Similarly, at $H=0.15, f=2.8716$ is the $35.85 \%$ decrease for the $f=4.4764$ of $\mathrm{Ge} / \mathrm{Si}$ at $H=0$. Furthermore, the 


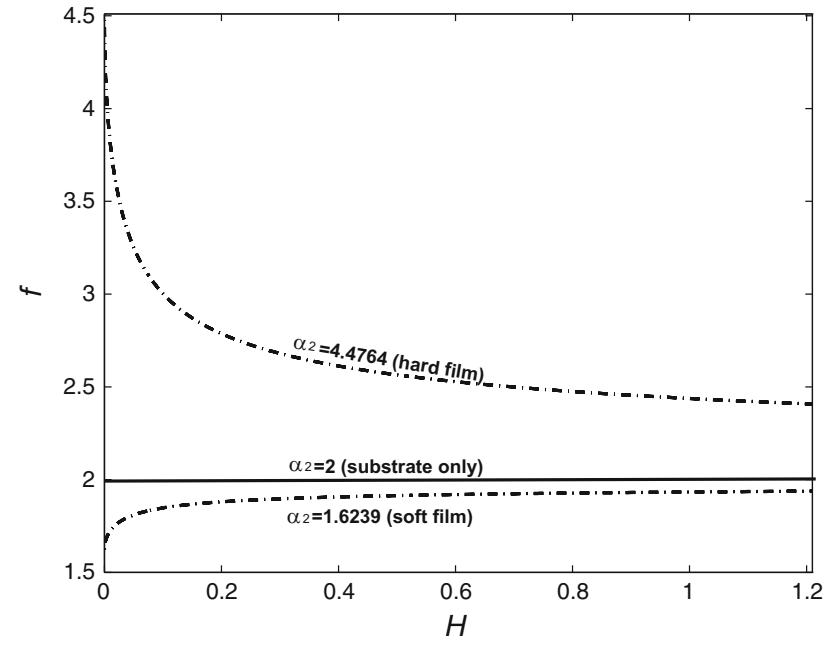

Fig. $5 H-F$ curve. Here $f=E^{\prime} / E_{i s}^{\prime}=\alpha_{3}+\left(\alpha_{2}-\alpha_{3}\right) \phi(x)$ is the (dimensionless) effective Young's modulus of the film/substrate heterostructure. The $\alpha_{2}=4.4764$ is the hard film scenario and $\alpha_{2}=$ 1.6239 is the soft film scenario. In both cases, as the indentation depth $(H)$ increases, the film effect decreases and the substrate effect becomes more important, which is the mechanism by which both curves approach $\alpha_{2}=2$. Physically, $\alpha_{2}=2$ corresponds to the substrate-only scenario

formation of intermolecular bonds between the nanoscale film and substrate layers $[7,13,23]$ can enhance the substrate effect. Clearly, this rule of thumb cannot be accurate, especially when the difference between the Young's moduli of the film and substrate is large. The above conclusions also hold for $\phi(x)=\mathrm{e}^{-\Lambda x}$. Actually, the fitting parameter $\Lambda=0.8$ can be obtained by the least squares method of fitting $\phi_{g}(x)$ in the range of $10^{-4} \leqslant x \leqslant 1.4$, and the difference between $\mathrm{e}^{-\Lambda x}$ and $\phi_{g}(x)$ is less than $5 \%$ [19].

Figures 2, 3 and 4 present the direct problem of the film/substrate indentation. The $A-F$ and $H-F$ curves are given by Eqs. (13), (14) and (20). In the $A-F$ and $H-F$ curves, $\alpha_{1}$ needs to be updated as the contact depth/radius changes, which is given by Eq. (18) as $\alpha_{1}=\beta_{1} f /\left(\beta_{1}+f\right)$. In real applications of the film/substrate indentation tests, the inverse problem is encountered: Given the $A-F$ curve, how can the reduced Young's modulus $\left(\alpha_{2}\right)$ and thickness $(T)$ of the film be extracted? Equation (19) provides the mathematical foundation for our formulation of the inverse problem. The physical mechanism that the inverse problem can solve is reflected in Figs. 4 and 5: the $H-F$ and $H-$ $F$ (substrate effect) curves depend on the Young's moduli of film and substrate, indentation depth and film thickness. These parameters have different effects on the curves, and monotonic relations are formed. Here the two unknowns are the reduced modulus $\left(\alpha_{2}\right)$ and thickness $(T)$ of the film, while the reduced moduli of the indenter $\left(\beta_{1}\right)$ and substrate $\left(\alpha_{3}\right)$ are known and fixed quantities. The reduced modulus of the specimen/indenter system $\left(\alpha_{1}\right)$ and contact radius $(A)$ are known and variable quantities. In an indentation test, $\alpha_{1}$ and $A$ are extracted from the experimental data in conjunction with the contact models or stiffness models as discussed above. Theoretically, an indentation test can provide infinite sets of $\alpha_{1}$ and $A$. However, there are only two unknowns, and thus only two different sets of $\alpha_{1}$ and $A$ are needed to solve the inverse problem. Again, because the indenter and substrate here are made of the same material, $\beta_{1}=\alpha_{3}=2$ are fixed. Now, for the $\mathrm{Ge} / \mathrm{Si}$ with $\alpha_{2}=1.6239$ and $T=1.5$, suppose that the two sets of the exact $\left(\alpha_{1}, A\right)$ values are extracted from the $A-F$ curve as $\left(\alpha_{1}, A\right)=(0.9235,0.5)$ and $(0.9732,3.0)$, which are then substituted into Eq. (19) as follows:

$$
\begin{aligned}
\mathcal{F}\left(\beta_{1}, \alpha_{1}, \alpha_{3}, A, \alpha_{2}, T\right) & =\mathcal{F}\left(2,0.9235,2,0.5, \alpha_{2}, T\right) \\
& =-0.284-\left(\alpha_{2}-2\right) \phi\left(\frac{0.5}{T}\right)=0, \\
\mathcal{F}\left(\beta_{1}, \alpha_{1}, \alpha_{3}, A, \alpha_{2}, T\right) & =\mathcal{F}\left(2,0.9732,2,3.0, \alpha_{2}, T\right) \\
& =-0.1044-\left(\alpha_{2}-2\right) \phi\left(\frac{3.0}{T}\right)=0 .
\end{aligned}
$$

Equation (21) provides two nonlinear equations for solving the two unknowns of $\alpha_{2}$ and $T$. The two unknowns are solved by the Newton-Raphson method [43], which yields the exact solutions of $\left(\alpha_{2}, T\right)=(1.6239,1.5)$. Here, $\phi(x)=\phi_{g}(x)$ is used. Again, the $A-F$ curve obtained from one indentation test can provide infinite sets of $\left(\alpha_{1}, A\right)$. If two different sets of $\left(\alpha_{1}, A\right)=(0.9416,1.0)$ and $(0.9559,1.5)$ are (arbitrarily) taken, the following equation set is obtained:

$$
\begin{aligned}
\mathcal{F}\left(\beta_{1}, \alpha_{1}, \alpha_{3}, A, \alpha_{2}, T\right) & =\mathcal{F}\left(2,0.9416,2,1.0, \alpha_{2}, T\right) \\
& =-0.2207-\left(\alpha_{2}-2\right) \phi\left(\frac{1.0}{T}\right)=0, \\
\mathcal{F}\left(\beta_{1}, \alpha_{1}, \alpha_{3}, A, \alpha_{2}, T\right) & =\mathcal{F}\left(2,0.9559,2,1.5, \alpha_{2}, T\right) \\
& =-0.1689-\left(\alpha_{2}-2\right) \phi\left(\frac{1.5}{T}\right)=0 .
\end{aligned}
$$

Again, the exact solutions of $\left(\alpha_{2}, T\right)=(1.6239,1.5)$ are obtained. As long as two arbitrarily and different sets of $\left(\alpha_{1}, A\right)$ are provided, the inverse problem as formulated in Eq. (21) or (22) always yields the exact solution for the Young's modulus and thickness of the film. Furthermore, because the initial guess for $\left(\alpha_{2}, T\right)$ in the Newton-Raphson method is required [43], formulation of the inverse problem as Eq. (21) is advantageous because it is insensitive to the initial guesses. Initial guesses of $\left(\alpha_{2}, T\right)=(1.99,2.1)$ and $(3.99,1.1)$ are used, both of which lead to the exact solution of $\left(\alpha_{2}, T\right)=(1.6239,1.5)$.

With the exact values of $\alpha_{1}$ and $A$ supplied by an indentation test, the exact solution of $\left(\alpha_{2}, T\right)$ is obtained for the inverse problem as formulated by Eqs. (21) and (22). However, the presence of errors is unavoidable in real tests. There are five major sources of errors in the $A-F$ curves. First, 
it is difficult to determine the initial contact point, especially when a sample is soft [20] or very thin [7]. This directly impacts the measurement of $H$ and thus $A$. Second, the indenter stiffness and shape calibration directly affects the measurement/interpretation of $F[7,33]$. Because the stiffness calibration involves a displacement measurement [7], displacement measurement error is a primary cause of calibration error. Third, there is a difference between the measured indenter displacement $(h)$ and the contact depth $\left(h_{c}\right)$ due to sink-in and pile-up [14,36,39], as discussed above. The fourth major error is caused by different contact models. As shown in Figs. 2 and 3, different contact models result in different $A-F$ curves. For a given data set of $(A, F)$, different contact models extract different $\alpha_{1}$, as seen in Eqs. (15) and (16). Furthermore, the contact radius $(A)$ is actually an ill-defined concept in different contact models [44]. For example, at the contact boundary, the contact stress of both the Hertz and DMT models is zero, while that of the JKR model is an infinite tensile stress [28,30]. The fifth source of error is the substrate effect model of Eqs. (9) and (11), wherein the effective film thickness cannot be accurately determined [16]. An effective means of addressing the first two sources of error is by improving the displacement resolution. Nowadays, indentation instruments such as the atomic force microscope have achieved a resolution of $0.1 \AA$ [7]. The third source of error is addressed by adding fitting parameters, as embodied in Eq. (7), to account for the effects of sink-in and pile-up. Errors caused by the different models can be mitigated by making deeper indentations or through the use of the contact stiffness model. As seen in Fig. 3, the slopes $(\mathrm{d} F / \mathrm{d} H)$ of the three contact models are (almost) the same when the indentation depth is relatively large. Therefore, if Eq. (6) or (7) is used to extract $E^{*}\left(\alpha_{1}\right)$, there will be little to no difference among the different contact models. As mentioned above, the pull-off force and thus the work of adhesion are subject to relatively large measurement fluctuations. The contact stiffness is much less prone to such fluctuations, and the contact stiffness model of Eq. (6) or (7) is recommended for the shallow indentation scenario. The fifth source of error can be lessened by shallow indentation with low compressive stress to reduce effects such as densification [16], indenter volume occupation [19] and plastic deformation [15].

The robustness of the above inverse problem-solving method is studied by inputting the (arbitrarily) erroneous data. One erroneous data set is input as $\left(\alpha_{1}, A\right)=(0.8855$, $0.56)$ and compared with the exact values of $\left(\alpha_{1}, A\right)=$ $(0.9235,0.50)$ as given above, with $\alpha_{1}$ and $A$ having the errors of $-4 \%$ and $12 \%$. The other erroneous data set is input as $\left(\alpha_{1}, A\right)=(0.9134,1.08)$ and compared with the above exact values of $\left(\alpha_{1}, A\right)=(0.9416,1.00)$, with $\alpha_{1}$ and $A$ having the errors of $-3 \%$ and $8 \%$. From the erroneous inputs, the following equations are obtained:

$$
\begin{aligned}
\mathcal{F}\left(\beta_{1}, \alpha_{1}, \alpha_{3}, A, \alpha_{2}, T\right) & =\mathcal{F}\left(2,0.8855,2,0.56, \alpha_{2}, T\right) \\
& =-0.4109-\left(\alpha_{2}-2\right) \phi\left(\frac{0.56}{T}\right) \\
& =0, \\
\mathcal{F}\left(\beta_{1}, \alpha_{1}, \alpha_{3}, A, \alpha_{2}, T\right) & =\mathcal{F}\left(2,0.9134,2,1.08, \alpha_{2}, T\right) \\
& =-0.3188-\left(\alpha_{2}-2\right) \phi\left(\frac{1.08}{T}\right) \\
& =0 .
\end{aligned}
$$

With the initial guess of $\left(\alpha_{2}, T\right)=(2.1,1.8)$, Eq. (23) provides the solution of $\left(\alpha_{2}, T\right)=(1.4601,1.6732)$. Compared with the exact solution of $\left(\alpha_{2}, T\right)=(1.6239,1.50)$, the error of $\alpha_{2}$ is $-10.08 \%$ and the error of $T$ is $11.55 \%$. Further study shows that the error of $T$ is much more sensitive to the input errors than that of $\alpha_{2}$. For example, the following two sets of $\left(\alpha_{1}, A\right)=(0.8773,0.56)$ and $(0.9055,1.08)$ are input, and the following equations are then obtained:

$$
\begin{aligned}
\mathcal{F}\left(\beta_{1}, \alpha_{1}, \alpha_{3}, A, \alpha_{2}, T\right) & =\mathcal{F}\left(2,0.8773,2,0.56, \alpha_{2}, T\right) \\
& =-0.4372-\left(\alpha_{2}-2\right) \phi\left(\frac{0.56}{T}\right) \\
& =0, \\
\mathcal{F}\left(\beta_{1}, \alpha_{1}, \alpha_{3}, A, \alpha_{2}, T\right) & =\mathcal{F}\left(2,0.9055,2,1.08, \alpha_{2}, T\right) \\
& =-0.3454-\left(\alpha_{2}-2\right) \phi\left(\frac{1.08}{T}\right) \\
& =0 .
\end{aligned}
$$

Compared with the previous erroneous input data of $\left(\alpha_{1}, A\right)$ $=(0.8855,0.56)$ and $(0.9134,1.08)$, the errors of $\alpha_{1}$ change from $-4 \%$ to $-5 \%$ and from $-3 \%$ to $-4 \%$, respectively. The errors of $T$ are unchanged at $12 \%$ and $8 \%$, respectively. Equation (24) provides the solution of $\left(\alpha_{2}, T\right)=$ $(1.44,1.8895)$. Now the corresponding error of $\alpha_{2}$ and $T$ is $-11.32 \%$ and $25.97 \%$. As the input errors of $\left(\alpha_{1}, A\right)$ increase, the error in both $\alpha_{2}$ and $T$ increases, and the error associated with $T$ increases more dramatically. Here, the errors of $T(12 \%, 8 \%)$ and $\alpha_{1}(-5 \%,-4 \%)$ are actually relatively large. The current state-of-the-art indentation instrument is capable of sub-angstrom displacement resolution, and the errors can be controlled within 5\%, even when the indentation depth is only several ångströms [7].

In this study, the effective reduced Young's modulus and thickness of the film are the only two unknowns to be determined by the inverse problem. To solve the inverse problem, only two different sets of $\left(\alpha_{1}, A\right)$ extracted from the $A-F$ curve need to be supplied, and this scenario is preferred by experimentalists [17]. There are infinite sets of $\left(\alpha_{1}, A\right)$ that can be extracted from the $A-F$ curve by one continuous indentation test. With the inverse problem formulation framework of Eq. (21), more unknowns such as the reduced Young's modulus of the substrate $\left(\alpha_{3}\right)$ can be included, which 
implies a more general and wider application of this inverse problem-solving method [45]. For soft sample indentations, the effects of finite strain, viscoelasticity and plastic dilatation etc. [25] can significantly complicate the accurate interpretation of the $A-F$ curve. Linear elasticity is assumed in the model, and therefore extreme caution should be exercised when applying the above inverse problem-solving method to a soft sample indentation test.

\section{Summary}

In this work, the inverse problem of determining the reduced Young's modulus and thickness of the film in a film/substrate indentation test is formulated and solved. The effective reduced Young's modulus of the film/substrate heterostructure depends on the reduced Young's moduli of the film and substrate, along with the indentation depth. The change in the effective Young's modulus of the film/substrate is reflected in both the $a-P(A-F)$ curve of the indentation test and the substrate effect models with analytical function forms. By equating the effective reduced Young's moduli extracted from the experimental $a-P$ curve at two different contact radii with those predicated on the substrate effect model at the same contact radii, the inverse problem is formulated and solved by the Newton-Raphson method. The indentation instrument, physical phenomena such as sink-in and pile-up, and the use of different contact and substrate effect models can all impact the measurement and interpretation of the $a-P$ curves, which leads to errors. The robustness of the inverse problem-solving method is demonstrated upon inputting the erroneous data.

Acknowledgements This research was supported by the National Natural Science Foundation of China (11772335, 21622304, 61674045, and 21203038) and by the Ministry of Science and Technology (MOST) of China (2016YFA0200700). Z.H. Cheng was supported by the Distinguished Technical Talents Project and the Youth Innovation Promotion Association of Chinese Academy of Sciences.

\section{References}

1. Britnell, L., Ribeiro, R.M., Eckamm, A., et al.: Strong light-matter interactions in heterostructure of atomically thin film. Science $\mathbf{3 4 0}$, 1311-1314 (2013)

2. Bonaccorso, F., Colombo, L., Yu, G., et al.: Graphene, related two dimensional crystals, and hybrid systems for energy conversion and storage. Science 347, 1246501 (2015)

3. Jiang, J.: Graphene versus $\mathrm{MoS}_{2}$ : a short review. Front. Phys. 10, 106801 (2015)

4. Liu, X., Galfsky, T., Sun, A., et al.: Strong light-matter coupling in two dimensional atomic crystals. Nat. Photon. 9, 30-34 (2015)

5. Hong, X., Kim, J., Shi, S., et al.: Ultrafast charge transfer in atomically thin $\mathrm{MoS}_{2} / \mathrm{WS}_{2}$ heterostructure. Nat. Nanotechnol. 9, 682-686 (2014)
6. Come, J., Xie, Y., Naguib, M., et al.: Nanoscale elastic changes in $2 \mathrm{D} \mathrm{Ti}_{3} \mathrm{C}_{2} \mathrm{~T}_{x}$ (MXene) pseudocapacitive electrode. Adv. Energy Mater. 6, 1502290 (2016)

7. Gao, Y., Kim, S., Zhou, S., et al.: Elastic coupling between layers in two-dimensional materials. Nat. Mater. 14, 714-720 (2015)

8. Zhu, Y., Xu, F., Qin, Q., et al.: Mechanical properties of vaporliquid-solid synthesized silicon nanowires. Nano Lett. 9, 39343939 (2009)

9. Lee, C., Wei, X., Kyser, J.W., et al.: Measurement of the elastic properties and intrinsic strength of monolayer graphene. Science 321, 385-388 (2008)

10. Koenig, S.P., Boddeti, N.G., Dunn, M.L., et al.: Ultrastrong adhesion of graphene membranes. Nat. Nanotechnol. 6, 543-546 (2011)

11. Zhang, Y.: Large deflection of clamped circular plate and accuracy of its approximate analytical solutions. Sci. China Phys. Mech. Astron. 59, 624602 (2016)

12. Zhang, Y., Liu, Y.: The Mexican hat effect on the delamination buckling of a compressed thin film. Acta Mech. Sin. 30, 927-932 (2014)

13. Zhang, J., Yuan, B., Cheng, P., et al.: Exceptionally stiff twodimensional molecular crystal by substrate-confinement. ACS Nano 11, 11425-11431 (2014)

14. Saha, R., Nix, W.D.: Effects of the substrate on the determination of thin film mechanical properties by nanoindentation. Acta Mater. 50, 23-38 (2002)

15. Lim, Y.Y., Chaudhri, M.M., Enomoto, Y.: Accurate determination of the mechanical properties of thin aluminum films deposited on sapphire flats using nanoindentation. J. Mater. Res. 14, 2314-2327 (1999)

16. Li, H., Vlassak, J.J.: Determining the elastic modulus and hardness of an ultra-thin film on a substrate using nanoindentation. J. Mater. Res. 24, 1114-1125 (2009)

17. Reed, J.L., Dean, J., Aldrich-Smith, G., et al.: A methodology for obtaining plasticity characteristics of metallic coatings via instrumented indentation. Int. J. Solids Struct. 80, 128-136 (2016)

18. Bull, S.J.: Nanoindentation of coatings. J. Phys. D Appl. Phys. 38, R293-R413 (2005)

19. Menčik, J., Munz, D., Quandt, E., et al.: Determination of elastic modulus of thin layers using nanoindentation. J. Mater. Res. 12, 2475-2484 (1997)

20. Dimitriadis, E.K., Horkay, F., Maresca, J., et al.: Determination of elastic moduli of thin layers of soft material using the atomic force microscope. Biophys. J. 82, 2798-2810 (2002)

21. Tetard, L., Passian, A., Farahi, R.H., et al.: Spectroscopy and atomic force microscopy of biomass. Ultramicroscopy 110, 701707 (2010)

22. Elder, R.M., Neupane, M.R., Chantawansri, T.: Stacking order dependent mechanical properties of graphene/ $\mathrm{MoS}_{2}$ bilayer and trilayer heterostructure. Appl. Phys. Lett. 107, 073101 (2015)

23. Zhang, J., Cheng, P., Yuan, B., et al.: Real-space identification of intermolecular bonding with atomic force microscopy. Science 342, 611-614 (2013)

24. Greenwood, J.A., Barber, J.R.: Indentation of an elastic layer by a rigid cylinder. Int. J. Solids Struct. 49, 2962-2977 (2012)

25. Needleman, A., Tvergaard, V., van der Giessen, E.: Indentation of elastically soft and plastically compressible solids. Acta Mech. Sin. 31, 473-480 (2015)

26. Hertz, H.: Über die Berührung fester elasticscher Körper (On the contact of elastic solids). J. rein und angwandte mathematik 92, 156-171 (1882). (For English translation see Miscellaneous Papers by H. Hertz, Eds. Jones and Schott, Macmillan, London, UK, 1896)

27. Derjaguin, B.V., Muller, V.M., Toporov, Y.P.: Effect of contact deformation on the adhesion of particles. J. Colloid Interface Sci. 53, 314-326 (1975)

28. Zhang, Y.: Transitions between different contact models. J. Adhes. Sci. Technol. 22, 699-715 (2008) 
29. Johnson, K.L., Kendall, K., Roberts, A.D.: Surface energy and the contact of elastic solids. Proc. R. Soc. Lond. A 324, 301-313 (1971)

30. Zhang, Y.: Adhesion map of spheres: effects of curved contact interface and surface interaction outside contact region. J. Adhes. Sci. Technol. 25, 1435-1464 (2011)

31. Tabor, D.: Surface force and surface interactions. J. Colloid Interface Sci. 58, 2-13 (1977)

32. Johnson, K.L., Greenwood, J.A.: An adhesion map for the contact of elastic spheres. J. Colloid Interface Sci. 192, 326-333 (1997)

33. Doerner, M.F., Nix, W.D.: A method for interpreting the data from depth-sensing indentation instruments. J. Mater. Res. 1, 601-609 (1986)

34. Pharr, G.M., Oliver, W.C., Brotzen, F.R.: On the generality of the relationship among contact stiffness, contact area, and elastic modulus during indentation. J. Mater. Res. 7, 613-617 (1992)

35. Carpick, R.W., Ogletree, D.F., Salmeron, M.: Lateral stiffness: a new nanomechanical measurement for the determination of shear strengths with friction force microscopy. Appl. Phys. Lett. 70, 1548-1550 (1997)

36. Oliver, W.C., Pharr, G.M.: Measurement of hardness and elastic modulus by instrumented indentation: advances in understanding and refinements to methodology. J. Mater. Res. 19, 3-20 (2004)

37. Hui, C.Y., Lin, Y.Y., Baney, J.M., et al.: The accuracy of the geometric assumptions in the JKR (Johnson-Kendall-Roberts) theory of adhesion. J. Adhes. Sci. Technol. 14, 1297-1319 (2000)

38. Xu, D., Ravi-Chandar, K., Liechti, K.M.: On scale dependence in friction: transition from intimate to monolayer-lubricated contact. J. Colloid Interface Sci. 318, 507-519 (2008)
39. Zhang, Y.: Extracting nanobelt mechanical properties from nanoindentation. J. Appl. Phys. 107, 123518 (2010)

40. Cheng, Y., Cheng, C.: Relationships between hardness, elastic modulus, and work of indentation. Appl. Phys. Lett. 73, 614-616 (1998)

41. Gao, H., Chiu, S., Lee, J.: Elastic contact versus indentation modeling of multi-layered materials. Int. J. Solids Struct. 29, 2471-2492 (1992)

42. Constantinescu, A., Korsunsky, A.M., Pison, O., et al.: Symbolic and numerical solution of the axisymmetric indentation problem for a multilayered elastic coating. Int. J. Solids Struct. 50, 27982807 (2013)

43. Press, W.H., Teukolsky, S.A., Vetterling, W.T., et al.: Numerical Recipes in Fortran, 2nd edn. Cambridge University Press, Cambridge (1992)

44. Greenwood, J.A.: Adhesion of elastic spheres. Proc. R. Soc. Lond. A 453, 1277-1297 (1997)

45. Zhang, Y., Zhao, Y.P., Cheng, Z.: Determining the layers? Youngs moduli and thickness from the indentation of a bilayer structure. J. Phys. D Appl. Phys. 51, 065305 (2018) 